\title{
El lenguaje de los niños ciegos y la cuestión de los rasgos autistas
}

\author{
Miguel Pérez Pereira' \\ Universidad de Santiago de Compostela
}

Hobson et al. (Brown, Hobson, Lee y Stevenson, 1997; Hobson, Brown, Minter y Lee, 1997) han investigado la similitud entre el autismo y la ceguera congénita. En este artículo se revisa la evidencia empírica a favor de la existencia de rasgos autistas en el lenguaje de los niños ciegos de nacimiento, a la vez que se aportan nuevos datos de investigaciones del autor. Se centra el interés en (a) el uso de los pronombres personales, (b) el uso de imitaciones y habla formuláica, (c) el uso egocéntrico del lenguaje, y (d) la capacidad de iniciar conversaciones y de contingencia conversacional. El análisis pone en cuestión la supuesta similitud entre los niños ciegos y los autistas, además parece refutar la teoría de Hobson.

Palabras claves: desarrollo del lenguaje, niños ciegos, rasgos autistas, pronombres personales, imitaciones verbales, capacidad conversacional.

The language in blind children and the question about autistic-like features

Hobson et al. (Brown, Hobson, Lee y Stevenson, 1997; Hobson, Brown, Minter y Lee, 1997) have updated the issue of the similarities between autism and congenital blindness. In the present paper the empirical evidence in favor of the existence of autistic-like features in the language of blind children is reviewed, and new data from the author's research, are offered. The following topics are examined: (a) The use of personal pronouns, (b) the use of imitations and formulaic speech, (c) the egocentric use of speech, and (d) the capacity for iniciating conversations and for conversational contingency. The analysis puts into question the similarity between blind children and autistic children, and seems to disconfirm Hobson's theory.

Key words: language development, blind children, autistic-like features, personal pronouns, verbal imitations, conversational capacity.

1 Doctor en psicología por la Universidad de Santiago de Compostela. Es catedrático de psicología evolutiva y de la educación en la Universidad de Santiago de Compostela. Director del Departamento de Psicología Evolutiva y de la Educación. A lo largo de su carrera ha investigado la adquisición y desarrollo del lenguaje en la infancia, y la adquisición del lenguaje en niños ciegos. Correo eJectrónico: pereira@usc.es. 

El presente estudio trata acerca de un tema que ha recobrado un gran interés recientemente: me refiero al tema de la existencia de rasgos autistas en el lenguaje de los niños ciegos. Es cierto que hace ya bastantes años, autores de orientación psicoanalítica como Keeler (1957), Fraiberg y Freedman (1964), Burlingham (1965), Nagera y Colonna (1965), Fay (1973), Fraiberg (1977) o Willis (1979) sugirieron que algunas características del lenguaje de los niños autistas se encontraban también en el lenguaje de los niños ciegos. Entre dichas características estaba el uso incorrecto de pronombres personales, el elevado uso del habla imitativa o ecolalia, las rutinas verbales y el habla formulaica, la abundancia de lenguaje egocéntrico y la ausencia de conversaciones iniciadas por los niños ciegos. Algunos de estos autores relacionaron esos rasgos autistas del lenguaje de los niños ciegos con los problemas que la ausencia de visión produce en las relaciones personales entre los niños ciegos y sus madres y, como consecuencia, en el desarrollo que los niños ciegos tienen de su yo (Burlingham, 1965; Fraiberg y Freedmam, 1964; Nagera y Colonna, 1965; Wills, 1979). Probablemente, la versión de esta perspectiva que más influencia ha tenido ha sido la de Selma Fraiberg (1977; Fraiberg y Adelson, 1973) quien ha considerado que la falta de visión afecta al desarrollo de la imagen que los niños ciegos tienen de sí mismos y su auto-representación y, por tanto, al desarrollo de su yo. Estas dificultades en el desarrollo personal tienen a su vez consecuencias en el lenguaje de los niños ciegos, especialmente en el uso que hacen de los pronombres personales.

Muy recientemente, Peter Hobson y sus colegas han puesto de moda la cuestión de las similitudes entre el autismo y la ceguera congénita (Brown, Hobson, Lee y Stevenson, 1997; Hobson, 1993a, 1993b; Hobson, Brown, Minter y Lee, 1997; Minter, Hobson y Bishop, 1998). Hobson et al. han indicado que "hay algo especial en la ceguera congénita que predispone al síndrome del autismo de manera parcial o total" (Brown, Hobson, Lee y Stevenson, 1997, p. 701), y han tratado de dar una explicación 
de esas similitudes más a tono con teorías actuales. En su teoría, Hobson le da una relevancia especial a la percepción de las actitudes emocionales de los otros hacia la realidad exterior para el desarrollo del yo y de otros yoes, y para el desarrollo de una teoría de la mente; o, para decirlo con otras palabras, para la comprensión de la mente y de los procesos mentales de los otros y de uno mismo, entre los que se encuentran los deseos, las intenciones, los conocimientos y las creencias. Pero, además de la capacidad de percepción de las actitudes de los otros, es necesaria también una capacidad de empatizar con el otro, generalmente la madre o el padre durante los primeros meses de vida. Esta capacidad para captar las reacciones emocionales de los otros e identificarse con ellas aparece ya en comportamientos muy bien conocidos como el de la llamada referencia social, hacia los 10 meses de edad. Pues bien, para Hobson y sus colaboradores, los niños ciegos, al igual quẻ los autistas, tendrán una gran dificultad para desarrollar una teoría de la mente. En el caso de los niños ciegos esa dificultad radica especialmente, aunque no sólo, en la imposibilidad de observar las reacciones emocionales de los otros hacia aspectos del mundo externo; mientras que para los niños autistas, la dificultad radica en su incapacidad para empatizar con los otros y para diferenciar sus actitudes de las de los demás. Sea como fuere, el hecho es que los niños ciegos y los autistas desarrollarán una sintomatología parecida, una psicopatología evolutiva semejante, según Hobson (Hobson, 1993a, 1993b; Brown, Hobson, Lee y Stevenson, 1997; Hobson, Brown, Minter y Lee, 1997; Minter, Hobson y Bishop, 1998).

Entre esas semejanzas alegadas hay una serie de rasgos comportamentales y cognitivos, como las estereotipias comportamentales, las limitaciones y el retraso en el juego simbólico o las dificultades en el desarrollo de una teoría de la mente, que no voy a discutir aquí, y también hay una serie de rasgos del lenguaje, que son aquellos en los que centraré mi exposición. Todos estos rasgos llamados autistas del lenguaje de los niños ciegos proceden de descripciones realizadas por otros autores $y$ aceptadas por Hobson y colegas. Entre dichos rasgos están los siguientes: 
El lenguaje de los niños ciegos y la cuestión de los rasgos autistas

1. Uso tardío e incorrecto de los pronombres personales, y, en general, de los términos deícticos de referencia personal (incluyendo también a pronombres posesivos y adjetivos posesivos) (Andersen, Dunlea y Kekelis, 1984; Dunlea, 1989; Fraiberg, 1977; Fraiberg y Adelson, 1973; Erin, 1990; Warren, 1994).

2. Abundancia inusual de imitaciones y ecolalia en el habla (Andersen, et al., 1984, 1993; Burlingham, 1965; Dunlea, 1989; Fay, 1973; Keeler, 1957; Peters, 1987, 1994; Urwin, 1984a, 1984b; Wills, 1979).

3. Elevada frecuencia de estereotipias verbales, rutinas o habla formulaica (Andersen et al., 1984, 1993; Dunlea, 1989; Keeler, 1957; Peters, 1994).

4. Uso egocéntrico del lenguaje, que es usado en referencia a sus propias acciones o a los objetos que está manipulando, pero no para describir propiedades externas de los objetos o de las acciones realizadas por alguna otra persona (Andersen, et al., 1984, 1993; Dunlea, 1984, 1989).

5. Escasa iniciación de conversaciones y participación no contingente en la conversación (Kekelis y Andersen, 1984; Kekelis y Printz, 1996; Moore y McConachie, 1994).

A continuación revisaré la base empírica en que se fundamenta la asimilación de ceguera y autismo. Pero antes de eso quisiera solamente apuntar unas cuantas consideraciones de tipo teórico y metodológico sobre la concepción de Hobson y sobre los estudios del desarrollo del lenguaje en los que se apoya.

La primera es que hay otras perspectivas teóricas sobre el desarrollo de la teoría de la mente diferentes de la de Hobson, y que no suponen que los individuos ciegos deban ver mermada su capacidad para comprender los procesos mentales y construir una teoría de la mente (TOM 'theory of mind'). Por ejemplo, Baron-Cohen (1995) ha indicado específicamente que los ciegos adultos participan perfectamente en relaciones sociales y pueden usar también términos mentales, como creer, imaginar, pensar, suponer, etc. en su lenguaje, cosas ambas de las que los autistas son incapaces. Este mismo autor ha señalado que los niños ciegos 
tampoco parecen tener deteriorada su capacidad de atención conjunta (SAM 'shared attention mechanism'), esencial para el desarrollo de una teoría de la mente, aún cuando puedan presentar un cierto retraso inicial de su capacidad de atención conjunto y ciertos rasgos autistas transitorios, nunca permanentes ni duraderos.

La segunda consideración es de tipo metodológico. Como anteriormente he señalado, Hobson ha dado por buenas ciertas descripciones del lenguaje de los niños ciegos realizadas por otros investigadores, y, en parte, en ellas basa el apoyo a su teoría de la similitud entre niños autistas y ciegos, sin embargo, desgraciadamente, una buena parte de las investigaciones realizadas sobre el desarrollo del lenguaje en los niños ciegos carecen de rigor metodológico (Pérez Pereira y ${ }^{\top}$ Conti-Ramsden, 1999). En muchas investigaciones se aportan como evidencia de lo que se dice unos cuantos ejemplos entresacados del habla de los niños o impresiones generales, pero no se aportan análisis cuantitativos ni cualitativos rigurosos, lo que hace difícil la verificación de los resultados y su contrastación con los de otros estudios. Por otra parte, las investigaciones transversales realizadas, generalmente han empleado corpus lingüísticos muy reducidos, además de las limitaciones que todo estudio transversal presenta para conocer el proceso de cambio. En cuanto a las investigaciones longitudinales, han utilizado muestras muy escasas, generalmente de no más de 5 ó 6 sujetos. Teniendo en cuenta la enorme heterogeneidad que presenta la población de niños ciegos, es muy arriesgado hacer generalizaciones a partir del estudio de unos cuantos sujetos. Para los que no están muy familiarizados con la problemática de los niños ciegos, es conveniente decir que la gran variabilidad que existe entre los niños ciegos se debe a múltiples factores, entre los que están las diferentes etiologías que pueden dar lugar a la ceguera, la existencia o no de trastornos asociados, la existencia de diferentes grados de agudeza visual aún dentro de lo que es la ceguera legal, las diferencias en cuanto al momento evolutivo en que comenzó la ceguera, o las diferencias 
El lenguaje de los niños ciegos y la cuestión de los rasgos autistas

en la educación y pautas de crianza de los niños ciegos, que pueden ser muy amplias.

En tercer lugar, los investigadores de la adquisición del lenguaje en los niños ciegos no han sido muy sensibles a las diferencias individuales, y en ellos ha predominado una visión bastante monolítica del desarrollo. Consistentemente con esta visión, cualquier diferencia encontrada entre los niños ciegos y los videntes ha sido interpretada como algo desviado, patológico o no normal. Por eso no se contempla la posibilidad de que ciertos rasgos del lenguaje de los niños ciegos, aparentemente iguales al de los niños con visión, puedan tener un significado diferente, ni tampoco se contempla que puedan existir diferentes rutas en el desarrollo del lenguaje. De manera semejante, tampoco se contempla que entre los niños ciegos pueda haber diferencias, y hay una tendencia a ofrecer una visión homogénea de su desarrollo lingüístico.

Finalmente, una buena parte de las investigaciones realizadas sobre la adquisición del lenguaje en los niños ciegos ha pasado por alto el hecho de que una misma forma lingüística puede cubrir funciones distintas dependiendo de su contexto de uso, y, a la inversa, dos tipos de producciones diferentes pueden cubrir una función semejante. Si aplicamos al tema de los rasgos autistas del lenguaje de los niños ciegos este principio de la no equivalencia automática de forma y función, el hecho de que los niños ciegos y los autistas produzcan expresiones aparentemente iguales, eso no quiere decir que sus funciones, o sus significados, sean idénticos en ambos casos. Por eso cualquier indagación de las similitudes entre el lenguaje de los niños ciegos y los autistas debe ir más allá de la primera analogía superficial o co-ocurrencia de fenómenos semejantes. Por fortuna, estas consideraciones no son aplicables a todos los estudios de la adquisición del lenguaje en los niños ciegos. Algunos investigadores han sostenido una visión diferente, y desde esta perspectiva, analizaré la evidencia empírica existente sobre los llamados rasgos autistas del lenguaje de los niños ciegos, para lo cual me apoyaré 
fundamentalmente en los datos de mi propia investigación que he realizado durante los 10 ó 12 últimos años.

\section{Términos de referencia personal}

La adquisición de los términos de referencia personal, y la de los pronombres personales en particular, ha sido considerada como uno de los rasgos más distintivos del lenguaje de los niños ciegos. Según unos cuantos investigadores que han tenido un elevado impacto en el campo, los niños ciegos producen muchos errores de inversión pronominal, como usar tú en vez de yo (Dunlea, 1989; Fraiberg, 1977; Fraiberg y Adelson, 1973; Peters, 1987). Además, los niños ciegos comienzan a usar los pronombres personales de una manera productiva más tarde que los niños videntes, después de los 3 años y medio. Hasta esa edad solamente emplean los pronombres de una manera sincrética o estereotipada, en frases hechas o fórmulas. Por otra parte, las dificultades en el uso de los pronombres personales son uno de los rasgos comportamentales más característicos de los niños autistas (Frith, 1989; Happé, 1994; Lord y Paul, 1997).

Como es bien sabido, los términos de referencia personal designan diferentes roles conversacionales, como hablante, oyente y no participante. Esas funciones conversacionales son cubiertas por la primera persona, la segunda persona y la tercera persona, respectivamente. Por eso, un dominio pleno de los términos de referencia personal requiere un conocimiento de su referencia cambiante. Su correcta comprensión y uso requiere que los niños puedan cambiar su perspectiva dependiendo del rol conversacional que están jugando. Se ha considerado que el cambio de referencia hace de los términos de referencia personal en particular, y de los términos deícticos en general, algo especialmente difícil de ser adquirido por los niños. Por tanto, parece que los niños que producen errores de inversión pronominal tienen dificultades en cambiar de perspectiva, tal como han sugerido Hobson (1993a) y Andersen et al. (1993). 
Como anteriormente he indicado, varios autores han señalado las similitudes existentes entre los niños ciegos y los autistas en cuanto al uso de los pronombres personales (Brown, Hobson, Lee y Stevenson, 1997; Fay, 1973; Hobson, 1993a, 1993b; Hobson, Brown, Minter y Lee, 1997). Sin embargo, la evidencia empírica de los problemas de los niños ciegos con los pronombres es relativamente débil, puesto que tanto Dunlea (1989) como Fraiberg (1977) solamente ofrecen como apoyo a sus afirmaciones sobre la inversión pronominal en los niños ciegos unos cuantos ejemplos de esos errores u observaciones anecdóticas. Como única información "cuantitativa", Dunlea (1989, pp. 83-84) afirma que "aproximadamente un 75\% de las referencias de Teddy (un niño ciego) a sí mismo implicaban errores pronominales". Como explicación del elevado porcentaje de inversiones pronominales Dunlea (1989) apela a la gran cantidad de imitaciones y habla estereotipado en el lenguaje de los niños ciegos (véase también Peters, 1987, para una explicación semejante).

Dado que no se ha aportado ningún análisis cuantitativo de las producciones pronominales de los niños ciegos, es fácil que se puedan introducir sesgos subjetivos en las interpretaciones de los investigadores. Precisamente para evitar ese problema se necesitan análisis cuantitativos y cualitativos detallados de la producción de pronombres, que estén basados en muestras representativas del lenguaje. Esto es precisamente lo que he intentado realizar en un trabajo reciente (Pérez-Pereira, 1999). En esta investigación he analizado todos los pronombres personales y posesivos, así como los adjetivos posesivos, producidos por 5 niños que variaban en su grado de visión. Las características de los niños y los períodos de tiempo durante los que fueron estudiados aparecen en el siguiente Cuadro. 


\section{Cuadro 1}

Características de los niños del estudio sobre referencia personal

\begin{tabular}{|c|c|c|c|c|c|}
\hline Características & Nino 1 & Niña 2 & Niño 3 & Niñe 4 & Niña 5 \\
\hline $\begin{array}{l}\text { Graboure } \\
\text { rision }\end{array}$ & $\begin{array}{l}\text { Visión parcial. } \\
\text { Legalmente ciego }\end{array}$ & Ciega & Ciego & Ciega & $\begin{array}{l}\text { Vidente } \\
\text { Hermana } \\
\text { gemela de } 4\end{array}$ \\
\hline $\begin{array}{l}\text { Patologia } \\
\text { Periodo } \\
\text { estudiado }\end{array}$ & $\begin{array}{l}\begin{array}{l}\text { Degeneración } \\
\text { coriorretiniana }\end{array} \\
1 ; 2.7 \text { a } 2 ; 1.5\end{array}$ & 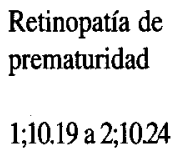 & $\begin{array}{l}\text { Retinopatía de } \\
\text { prematuridad } \\
2 ; 4.15 \text { a } 3 ; 5.27\end{array}$ & $\begin{array}{l}\text { Retinopatía de } \\
\text { prematuridad } \\
2 ; 5.28 \text { a } 3 ; 6.12\end{array}$ & $\begin{array}{l}- \\
2 ; 5.28 \mathrm{a} \\
3 ; 6.12\end{array}$ \\
\hline $\begin{array}{l}\text { Sestones: } \\
\text { grabadas: }\end{array}$ & 9 de 1 hora. & 12 & 13 & 11 & 11 \\
\hline
\end{tabular}

Los resultados hallados ponen de manifiesto que las primeras producciones de los pronombres personales de $1^{\mathrm{a}}$ y $2^{\mathrm{a}}$ persona de singular aparecen incluso antes de que los niños alcancen los 2 años de edad, y otras formas aparecen poco después, lo que indica que los niños ciegos no están retrasados si los comparamos con la niña vidente estudiada y con otros niños hablantes del español estudiados por otros investigadores (Hernández-Pina, 1984; Shum, Conde y Díaz, 1992; Pérez Pereira, Forján y García, 1996). Además, los niños ciegos emplearon las formas de referencia personal, especialmente las de $1^{a}$ y $2^{a}$ persona, con una gran frecuencia. No obstante, los resultados más interesantes y pertinentes son los que se refieren a la producción de los pronombres de $1^{\mathrm{a}}$ y $2^{\mathrm{a}}$ persona, que se muestran en la siguiente figura.

En esta gráfica, los pronombres personales y posesivos y adjetivos posesivos de $1^{\text {a }}$ y $2^{a}$ persona fueron clasificados según su uso fuese correcto, invertido o hubiese otros errores.

Los resultados hallados muestran claramente que la producción de inversiones pronominales no puede ser considerada como un rasgo común a todos los niños ciegos, sino solamente a una parte de ellos. De 


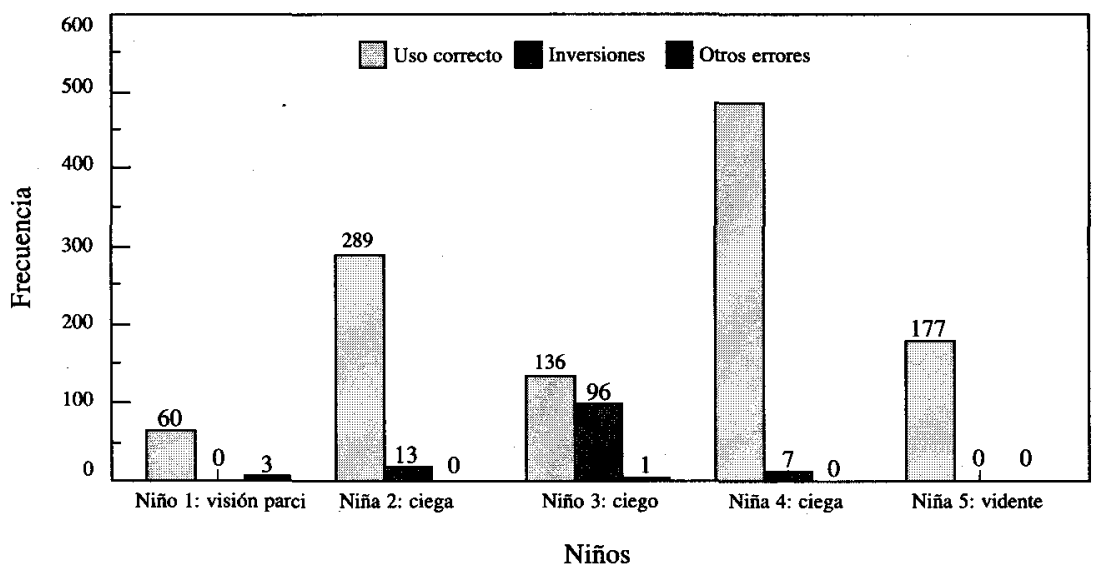

Figura 1. Uso de los pronombres de $1^{\circ}$ y $2^{\circ}$ personas

hecho, solamente el niño 3 presentaba un alto porcentaje de inversiones $(41,2 \%)$. Por el contrario, las otras dos niñas ciegas apenas producían errores de inversión $(4,4 \%$ y $1,5 \%$, la niña 2 y la niña 4 , respectivamente). Tanto el niño vidente como el que tenía un resto visual no produjeron ningún error de inversión, aunque sí otro tipo de errores. Sin embargo, es conveniente recordar que la producción de inversiones pronominales no es inusual en los niños videntes (Chiat, 1982; Dale y Crain-Thoreson, 1993). Algunos autores han defendido la hipótesis de que los niños ciegos producen inversiones al imitar las emisiones de otras personas, sin introducir el cambio preceptivo de persona (Dunlea, 1989; Fay, 1973; Peters, 1987). Para comprobar esta hipótesis se ha analizado tos contextos de uso en los que se producían los errores de inversión, entre los cuales está la producción de pronombres inmediatamente después de que el interlocutor acaba de producir uno (véase Pérez-Pereira, 1999 para una definición completa del sistema de categorías).

Los resultados indican que la imitación no puede explicar la mayoría de las inversiones producidas por el participante $3(29,9 \%)$, que era el que producía más inversiones, aunque sí puede ser útil para explicar las 
escasas inversiones producidas por los participantes 2 y 4 , como se muestra en la Figura 2.

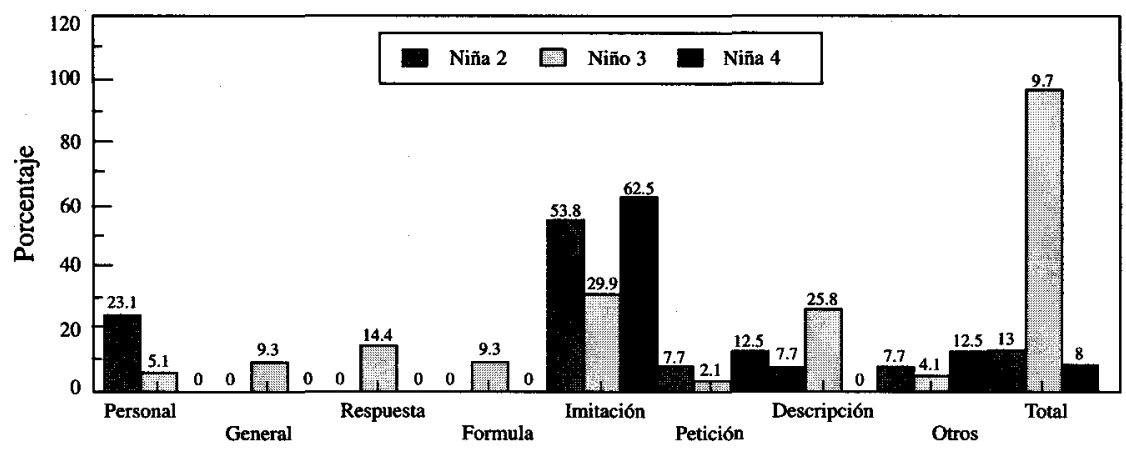

Tipos de errores de inversión

Figura 2. Contextos de producción de los errores de inversión (\%)

Recientemente Ann Peters (1999) ha obtenido datos que refrendan la insuficiencia de la imitación para explicar el fenómeno de la inversión pronominal. Al comparar las formas en que el padre de un niño ciego se refería a su hijo ciego Seth con las formas en que el propio Seth se refería a sí mismo, Peters halló una gran falta de correspondencia, que parece indicar que el niño no copiaba a su padre al referirse a sí mismo.

Volviendo a nuestros datos, como se puede apreciar en la Figura 2, el niño 3 producía muchos errores de inversión en emisiones como las siguientes: (a) como si el niño estuviese dirigiendo o describiendo su acción en curso, de la misma manera que haría un testigo de su acción (Personal: 5.1\%); (b) como si el niño estuviera dirigiéndose a sí mismo de una manera general o impersonal (General: 9.3\%); (c) contestando a una pregunta (Respuesta: 14.4\%); (d) describiendo las acciones o las intenciones de otros (Descripción: 25.8\%); o (e) reproduciendo rutinariamente el habla escuchada en una determinada situación (Fórmula: 9.3\%). Todas estas situaciones requieren del niño una habilidad para adoptar un punto de vista definido y para cambiar 
de perspectiva. Por tanto, estos resultados coinciden parcialmente con la sugerencia de Hobson (1993b; Lee, Hobson y Chiat, 1994) y de Andersen et al. (1993) de que los niños ciegos tienen dificultad para cambiar de perspectiva. Tomando en conjunto estos datos, parece que las inversiones pronominales se producen por una coincidencia de factores, y que no existe una explicación simple de esos errores. En cualquier caso, los errores de inversión no pueden ser considerados como un rasgo típico de todos los niños ciegos, ni tampoco, por tanto, como un rasgo común compartido con los niños autistas.

\section{Ecolalia, imitaciones y habla estereotipada}

Estas fueron algunas de las características que más tempranamente llamaron la atención de los investigadores. Autores clásicos, fundamentalmente de orientación psicoanalítica, remarcaron el carácter imitativo y estereotipado del lenguaje de los niños ciegos, y relacionaron ese rasgo con ciertas características deficitarias del desarrollo de su yo (ego) (Burligham, 1965; Keeler, 1957; Naggera y Colonna, 1965; Willis, 1979). Más tarde esas características fueron descritas también por otros muchos autores (Andersen et al., 1984, 1993; Dunlea, 1989; Kitzinger, 1984; Pérez-Pereira y Castro, 1992, 1997; Peters, 1987; Urwin, 1978, 1984a, 1984b). Sin embargo, aún cuando existe una coincidencia entre los investigadores en cuanto a la abundancia del habla estereotipada y las imitaciones en el lenguaje de los niños ciegos, existen diferencias en lo que se refiere a la interpretación de las imitaciones y las estereotipias. De una manera general, se puede decir que predominó una interpretación deficitaria de estas producciones hasta muy recientemente. En buena medida esto se debe a la utilización de un criterio restrictivo de lo que es imitación. La gran mayoría de investigadores que han estudiado el desarrollo de los niños ciegos o de los autistas (Tager-Flusberg y Calkins, 1990) ha adoptado un criterio muy restrictivo para definir lo que son imitaciones y repeticiones (entendidas como auto-repeticiones). Generalmente, solamente se han tenido en cuenta las copias exactas y completas o reducidas, a las que faltaba algún elemento, de las emisiones anteriores. Al hacer esto, los investigadores han 
excluido de los análisis aquellas producciones que son más creativas, como las imitaciones modificadas o las expandidas, y han tenido en cuenta solamente las producciones que son más mecánicas, como ha señalado acertadamente Catherine Snow (1983). Sin embargo, muchas emisiones que son una copia contienen una parte de la emisión modelo junto con algún cambio en alguno de sus componentes, o algún añadido. Por otra parte, el habla formulaica ha sido considerada como negativa, como una clase de habla sin significado, como la de los loros, sin ninguna utilidad para el desarrollo. Se ha adoptado un criterio no restrictivo para analizar las imitaciones y repeticiones, así como las rutinas verbales en una niña ciega y su hermana gemela con visión. Los resultados obtenidos fueron, en mi opinión, muy interesantes (Pérez-Pereira, 1994). En primer lugar se halló que la niña ciega usaba un número más elevado de rutinas $(7,91)$ que su hermana vidente, como refleja la Figura 3 correspondiente al tipo de producción.

Tipo de producción

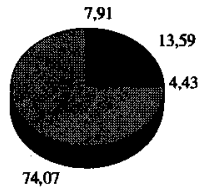

Niña ciega

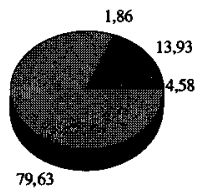

Niña vidente
Imitaciones y repeticiones:

Ajuste al modelo
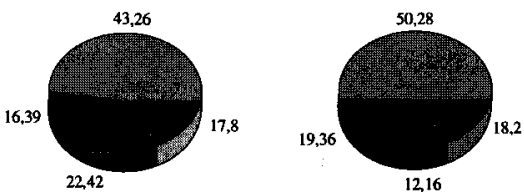

Niña ciega

Niña vidente

\begin{tabular}{|l}
$\square$ Imitación \\
Rutina
\end{tabular}

\begin{tabular}{|ll|}
\hline Exacta & $\square$ Reducida \\
Modificada & $\square$ Expandida \\
\hline
\end{tabular}

Figura 3. Lenguaje de las hermanas gemelas. 
Estos resultados coinciden con las descripciones del lenguaje de los niños ciegos que afirman que esos niños usan una gran cantidad de fórmulas, rutinas o habla estereotipado (Dunlea, 1989; Urwin, 1984). Ese tipo de producciones son consideradas como frases congeladas que son memorizadas en ciertos contextos y después reproducidas por los propios niños. Ann Peters (1994) ha sugerido de una manera convincente que el niño ciego que ella estudió aprendía esas frases formuláicas en contextos de interacción y actividad conjunta con su padre. Este lenguaje tan contextualizado, o fórmulas verbales, permitía al niño conocer la situación en la que estaba, e iniciar y promover la interacción social o el juego social con su padre. De esta manera, el habla formuláica compensa la falta de información visual, y ayuda al niño ciego a evitar el aislamiento social. En cierta forma, cada expresión formuláica formaba parte de un guión determinado para una actividad o situación específica, como el momento de la comida, el juego de construir torres con bloques, el de jugar a tirarse una pelota, etc. Por su parte, el padre utilizaba este lenguaje ajustado a situaciones específicas con la intención de andamiar las habilidades en desarrollo de su hijo y de promover su participación en actividades rutinarias.

Aún cuando las rutinas verbales o fórmulas se dan en contextos muy específicos al principio, posteriormente parece que los niños ciegos son capaces de generalizar su uso más allá de rutinas específicas a circunstancias similares, como Peters (1994) y Norgate, Lewis y Collis (1997) han mostrado. Por ejemplo, el niño ciego estudiado por Peters (1994) utilizó por primera vez las palabras on y off al encender y apagar (turn on y turn off) la grabadora; pero después utilizó esas palabras abriendo y cerrando un grifo (turn on y turn off), y cuando le ponían y le quitaban la ropa (put on y put off). Situaciones como esas indican que, además, las rutinas verbales son utilizadas por los niños ciegos para planificar y dirigir su propia actividad. Como Peters, acertadamente, ha afirmado "la falta del canal visual hace al niño ciego más dependiente del habla, como fuente de información acerca del mundo y como medio de interacción social, a la vez" (Peters, 1994, p. 199). 
El uso de expresiones formuláicas se apoya muy fuertemente en la capacidad de los niños ciegos para imitar y memorizar el habla de los adultos, lo que algunas veces da lugar a usos temporales de fórmulas que tienen sentido dentro de un contexto, pero que no son corectas desde el punto de vista sintáctico (Wilson y Peters, 1988). Más adelante, lo que fueron anteriormente frases congeladas pasan a ser analizadas y se convierten en habla productiva, tal como han señalado varios autores (Dunlea, 1989; Lieven, Pine y Baldwin, 1997; Mieczinkowski y Andersen, 1986; Pérez-Pereira, 1994; Peters, 1987, 1994; Pine y Lieven, 1993).

En la Figura 3, las dos niñas gemelas, la vidente y la ciega, no parece que tengan diferencias en el uso que hacen de imitaciones y repeticiones. Sin embargo, un análisis más detallado de estas emisiones (ajuste al modelo) reveló que la niña ciega utilizó con mayor frecuencia que su hermana vidente imitaciones y repeticiones modificadas $(22.42 \%)$, y que ambas hermanas utilizaron muchas imitaciones y repeticiones que eran reducidas o expandidas. Estas emisiones combinan una parte que es una copia de una emisión anterior con un elemento que se substituye (en el caso de las modificadas) o con nuevos elementos (en el caso de las expandidas).

Las imitaciones y repeticiones pueden servir para mantener una conversación o para realizar un acto de habla, y esas funciones pueden ser cubiertas por las imitaciones y repeticiones modificadas y expandidas, también. Sin embargo, cabe destacar que las imitaciones y repeticiones modificadas y expandidas pueden formar parte de una estrategia para analizar el lenguaje mediante la segmentación y substitución de algunas partes de la emisión o su expansión. Esta estrategia es utilizada por todos los niños, pero, probablemente, en una mayor medida por los niños ciegos.

Los siguientes son algunos ejemplos de lo explicado más arriba:

(1) La niña ciega y la vidente están merendando con su madre a $\operatorname{los} 2 ; 11,7$. 
Madre: Sandra no se mastica, te lo acabo de decir.

Niña vidente: ¿Que no se qué?

Niña vidente: ¿Que no se qué?

(Poco después)

Niña ciega: Andrea no se tiran las cosas.

En este ejemplo, la niña ciega está usando una estructura modelo (Nombre + no sé + PV) copiado del habla de su madre. En otros ejemplos, la niña modifica progresivamente su enunciado para expresar su intención de una manera más explícita. Lo que es interesante es observar cómo la niña está segmentando y substituyendo elementos poco a poco, como en los siguientes ejemplos:

(2) La niña ciega a los $3 ; 3,13$.

Niña ciega: Una trompeta cogí.

Niña ciega: Mira qué cogí.

Niña ciega: Mira lo que cogí.

(3) La niña ciega a los 2; 11,7 está jugando con una tabla de madera para encajar animales.

Niña ciega: Poner el camello a mí.

Niña ciega: Lo ponemos aquí

Niña ciega: Ponemos aquí el camello.

(Ejemplos tomados de Pérez-Pereira, 1994)

Algunos autores (Lleven, Pine y Baldwin, 1997; Pérez-Pereira, 1994; Peters, 1987; Pine y Lieven, 1993) han sugerido que la estrategia para analizar el lenguaje a la que me he referido anteriormente es típica de los niños que adoptan un estilo gestáltico, quienes tienden a "usar primero y analizar después" (Peters, 1987). Tradicionalmente se ha considerado que este estilo no era tan efectivo para aprender el lenguaje como el estilo analítico, pero Julian Pine y Elena Lieven (1993) han argumentado convincentemente que adoptar un estilo u otro no afecta a un mejor o peor aprendizaje del lenguaje, sino que simplemente son dos rutas diferentes que llevan al mismo resultado final. 
Desafortunadamente, esta forma de pensar no ha caracterizado a muchos de los investigadores que han estudiado el habla imitativa y estereotipado en los niños ciegos. Por tanto, parece que la función que las imitaciones y el habla formulaica tienen en los niños ciegos es diferente del uso que de ellas hacen los niños autistas, en los que, al parecer, no juegan ningún papel potenciador del proceso de adquisición (Lord y Paul, 1997).

\section{Habla egocéntrica}

Dunlea et al. (Andersen et al., 1984, 1993; Dunlea, 1984, 1989) han afirmado con rotundidad que los niños ciegos solamente usan el lenguaje para referirse a los objetos que están manipulando, o a las acciones que están realizando, pero que son incapaces de hacerlo para referirse a objetos externos y a acciones o acontecimientos en los que los niños mismos no están implicados. Dunlea (1989, p. 52) también ha afirmado que los niños ciegos usan los primeros términos relacionales (como mine, no, all-gone, o more: ' mío', 'no', 'se fue' o 'más') "como un medio para satisfacer sus propias necesidades, más que en relación con los estados dinámicos de las entidades". $\mathrm{Pa}$ rece claro desde esta concepción que los niños ciegos deben ser bastante parecidos a los autistas. Sin embargo, una vez más, Dunlea no realiza ningún análisis cuantitativo. Pero lo que es aún más importante es que esta caracterización del lenguaje de los niños ciegos como auto-centrado discrepa de lo que es relativamente común a los niños pequeños sin handicap visual. Algunos autores (Barrett, 1983) han hallado que los niños videntes, al comienzo de su desarrollo lingüístico, usan las palabras referidas a acciones como un acompañamiento de su actividad en curso o de las acciones que van a realizar inmediatamente. Posteriormente, usarán ese tipo de palabras con relación a las acciones realizadas por otros. Puesto que todos los niños estudiados por Dunlea (1989) tenían alrededor de 2 años al final de su estudio longitudinal, parece razonable suponer que no usasen muchas palabras de una manera descentrada. En otro estudio del lenguaje de los niños ciegos 
El lenguaje de los niños ciegos y la cuestión de los rasgos autistas

Cathy Urwin (1984a, 1984b) observó que un niño ciego de 2;3 años era capaz de hablar de las acciones y las posesiones de objetos de otras personas con tal de que esas acciones y objetos le fuesen familiares. Este hecho sugiere que, a medida que los niños ciegos crecen, superan las limitaciones iniciales en el uso de las palabras para acciones. Los datos que Fina Castro y yo hemos reunido en un estudio longitudinal sobre el desarrollo pragmático del habla de las dos niñas gemelas mencionadas (Castro y Pérez-Pereira, 1996; Pérez-Pereira y Castro, 1992, 1997) parecen dar crédito a esta idea. En dicho estudio analizamos el uso de un conjunto de categorías pragmáticas desde los $2 ; 5$ a los $5 ; 3$, en sesiones aproximadamente mensuales de observación de una hora de duración (por ej. Castro y Pérez Pereira, 1996; Pérez-Pereira y Castro, 1997 para una descripción detallada del procedimiento y categorías de análisis empleadas). Para simplificar los resultados, los datos se presentan agrupados en tramos de edad de 6 meses. Aunque nuestro interés principal no era el del análisis del habla egocéntrica, hay datos del uso de varias categorías pragmáticas por la niña ciega y su hermana con visión que son apropiados para analizar este problema.

Muy brevemente se mostrará la tendencia evolutiva observada en el uso de unas cuantas funciones pragmáticas por ambas niñas. Esas funciones eran solamente una parte de todas las funciones analizadas, por lo que los porcentajes de uso son relativos al total. En primer lugar se mostrará los resultados de una categoría, la de descripciones, que está muy estrechamente relacionada con el uso del habla descentrada. Esta categoría incluye la identificación de objetos, descripción de acontecimientos externos, descripción de características de los objetos, localizaciones de objetos, acciones y acontecimientos y tiempo de ocurrencia de acciones y acontecimientos. Los resultados hallados indican que la niña ciega era capaz de producir descripciones desde el comienzo del estudio, y que había un incremento constante en el uso de esta categoría, de tal manera que el patrón de uso de la niña ciega se aproximaba progresivamente al de la niña vidente. A continuación se 
presenta los resultados obtenidos por las dos niñas en dos categorías: informes internos y personal, que también aparecen en la Figura 4.

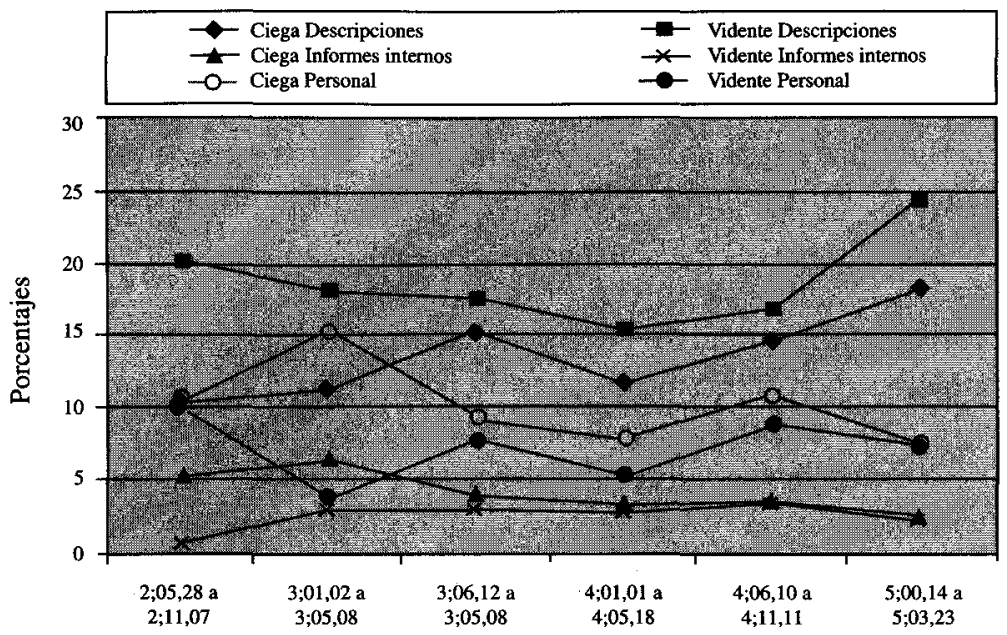

Tramos de edad

Figura 4. Porcentaje de uso de las categorías

La primera categoría está relacionada con la expresión de los propios deseos, y la segunda con la descripción de las acciones realizadas por uno mismo y la intención o determinación de realizar una acción inmediatamente. En resumen, estas dos categorías están muy relacionadas con los usos egocéntricos o autocentrados del lenguaje. Como se puede observar en la Figura 4, después de los 3;6 años las dos niñas mostraban una frecuencia de uso similar. Todos estos resultados sugieren que, a medida que la niña ciega crecía, usaba el lenguaje de una manera progresivamente menos centrada en sí misma. De cualquier forma, es lógico que los niños ciegos tengan más dificultades que los niños videntes para percibir las actividades realizadas por otros, los acontecimientos externos y los objetos distantes, y que, por consiguiente, hablen menos sobre ellos que los niños videntes. Sin embargo, eso se debe a 
una restricción de la experiencia que les es accesible y no a una incapacidad intrínseca para hacer descripciones. Es sintomático que de los ejemplos que ofrece Dunlea (1989) del habla de los niños ciegos de descripciones de acciones realizadas por otras personas, una buena parte se refieran a acciones que tenían un efecto sonoro (como eructar). Finalmente, me gustaría referirme al último de los rasgos autistas del lenguaje de los niños ciegos.

\section{Iniciación de conversaciones y contingencia conversacional}

Como se ha señalado anteriormente, varios investigadores han afirmado que los niños ciegos no inician conversaciones y tienden a realizar menos contribuciones contingentes en las interacciones conversacionales que los niños videntes. Sin embargo, la evidencia empírica de éstas afirmaciones proviene esencialmente del trabajo de Moore y McConachie (1994), que se basa en una muestra de lenguaje muy reducida, de solamente 15 minutos de conversación, obtenida de 16 niños, de los cuales 8 eran ciegos y 8 eran deficientes visuales. Los datos de Moore y $\mathrm{McCo}-$ nachie (1994) aparecen en el Cuadro 2, así como los del estudio longitudinal de Kekelis y Andersen (1984) con dos niños ciegos y dos videntes de menos de 2 años (los datos se ofrecen para dos tramos de edad).

Gina Conti-Ramsden y yo (Conti-Ramsden y Pérez-Pereira, 1999; Pérez-Pereira y Conti-Ramsden, en prensa) hemos llevado a cabo dos investigaciones para estudiar las interacciones conversacionales entre niños ciegos y sus madres, comparándolo con lo que ocurre con parejas en las que el niño o la niña no tiene déficit visual. En el primer estudio, los sujetos eran 3 niños ciegos y 1 niña vidente y sus madres durante dos períodos del desarrollo. El primer período iba desde los $2 ; 4$ a los $2 ; 10$, y el segundo desde los $2 ; 11$ a los 3;4. En el segundo estudio los sujetos eran una niña ciega, un niño con déficit visual, y una niña vidente, y sus respectivas madres. En este segundo estudio, los niños fueron estudiados entre $\operatorname{los} 1 ; 10$ y los 2;1 años. Como se puede apreciar nuestros sujetos eran un poco más mayores 


\section{Cuadro 2}

Proporción media de episodios conversacionales iniciados por el niño y por los padres según Kekelis y Aindersen (1984), y Moore y McConachie (1994)

\begin{tabular}{|c|c|c|c|c|c|c|}
\hline & Grupo & ciegos & Grupo de & ón parcial & Grupo & videntes \\
\hline & Madres & Niños & Madres & Niños & Madres & Niños \\
\hline $\begin{array}{l}\text { Kekelis y Andersen } \\
16-18 \text { meses }\end{array}$ & 77 & 23 & $\cdots$ & $-\cdots$ & 64 & 36 \\
\hline $\begin{array}{l}\text { Kekelis y Andersen } \\
19-22 \text { meses } \\
\text { Moore y McConachie } \\
18.2 \text { ciego / } 16.5 \text { V.P. }\end{array}$ & 75 & 25 & 38 & 62 & 62 & 38 \\
\hline
\end{tabular}

que los estudiados por Moore y McConachie (1994) y Kekelis y Andersen (1984). La virtud principal de nuestro estudio es que la muestra de lenguaje utilizada es mucho más representativa de las habilidades conversacionales de los niños y las madres estudiadas que la utilizada por Moore y McConachie (1994). Aunque la investigación que realizamos tenía unos objetivos más amplios, me gustaría ahora darles alguna información de los resultados que se refieren al porcentaje de conversaciones iniciadas por los niños y sus madres, así como a la longitud de los turnos conversacionales, que indica la contribución de los niños y las madres a la continuación de la conversación. Estos datos aparecen en los Cuadros 3 y 4 , respectivamente.

Como pueden observar, en el estudio 1 la diferencia entre la niña vidente y los niños ciegos es mínima, si tenemos en cuenta el porcentaje de inicios, $y$, particularmente, si comparamos a esta niña con su hermana gemela. Además, hay una importante variación en cada niño que no se puede atribuir a un estilo constante de cada sujeto. Es decir, existe una importante variación debida al contexto en él que tienen lugar las conversaciones. Por lo que respecta a la contingencia de las emisiones de los niños, la longitud media de las contribuciones de los niños ciegos a las unidades o episodios conversacionales no es inferior, 
El lenguaje de los niños ciegos y la cuestión de los rasgos autistas

Cuadro 3

Inicio de Conversaciones"

\section{Estudio 1}

\begin{tabular}{|c|c|c|c|c|c|c|c|}
\hline Niño & Alba & Javi & Sandra & Andrea & Javi & Sandra & Andrea \\
\hline${ }^{\circ}$ Visión & Ciego & Ciego & Ciego & Vidente & Ciego & Ciego & Vidente \\
\hline Edad & $2 ; 4-2 ; 10$ & $2 ; 4-2 ; 10$ & $2 ; 4-2 ; 10$ & $2 ; 4-2 ; 10$ & $2 ; 11-3 ; 4$ & $2 ; 11-3 ; 4$ & $2 ; 11-3 ; 4$ \\
\hline Madre & $61(55.5)$ & $148 \quad(50)$ & $22 \quad(49)$ & (40) & $66 \quad(58)$ & $115(45)$ & $50 \quad(39)$ \\
\hline Niño & 49 (44.5) & $148 \quad(50)$ & $23 \quad(51)$ & $(60)$ & $47 \quad(42)$ & $141 \quad(55)$ & $79 \quad(61)$ \\
\hline
\end{tabular}

\section{Estudio 2}

\begin{tabular}{llll}
\hline Niño & Alba & Eli & Noelia \\
${ }^{\circ}$ Visión & Ciego & V. parcial & Vidente \\
Edad & $1 ; 10-2 ; 1$ & $1 ; 10-2 ; 1$ & $1 ; 10-2 ; 1$ \\
Madre & $1351(67)$ & $850(56)$ & $686 \quad(52)$ \\
Niño & $668 \quad(33)$ & $679(44)$ & $637(48)$ \\
\hline
\end{tabular}

Nota. ${ }^{a}$ Porcentajes redondeados entre paréntesis.

\section{Cuadro 4}

Contingencia. Longitud Media de los Turnos

\begin{tabular}{lllll}
\hline Estudio 1 & & & & \\
\hline Niño & Alba & Javi & Sandra & A \\
${ }^{\circ}$ Visión & Ciego & Ciego & Ciego & V \\
Edad & $2 ; 4-2 ; 10$ & $2 ; 4-2 ; 10$ & $2 ; 4-2 ; 10$ & $2 ; 4$ \\
Madre & 33.2 & 5.03 & 3.97 & 3. \\
Niño & 19.23 & 2.44 & 3.08 & 3.9 \\
Total & 52.5 & 7.5 & 7.1 & 7.8 \\
& & & & \\
Estudio 2 & & & & \\
\hline Niño: & Alba & Eli & Noelia \\
o Visión: & Ciego & V.parcial & Vidente \\
Edad: & $1 ; 10-2 ; 1$ & $1 ; 10-2 ; 1$ & $1 ; 10-2 ; 1$ \\
Madre & 28.5 & 18.3 & 8.6 \\
Niño & 14 & 14.9 & 8.1 \\
Total & 42.5 & 33.2 & 16.8 \\
\hline
\end{tabular}


en términos generales, a la de la niña vidente, aunque estos resultados también sugieren que los esfuerzos de las madres de los niños ciegos para el mantenimiento de la conversación parecen ser mayores que los de la madre de la niña vidente; un resultado que es coincidente con el obtenido por Kekelis y Prinz (1996). En todo caso, si los niños ciegos estuviesen produciendo emisiones no contingentes, no hubiesen sido capaces de participar con tantos turnos por unidad conversacional. Los datos del estudio 2 son semejantes a los obtenidos en el estudio 1. Es interesante fijar nuestra atención en los resultados de los inicios de conversación en el estudio 2, porque el corpus sobre el cual se compara a los sujetos es semejante. Atendiendo a la frecuencia de inicios, vemos que no hay diferencias entre la niña ciega y la vidente, así como con el niño con deficiencia visual; pero si nos fijamos en los porcentajes, vemos que el porcentaje de inicios de conversaciones de la niña ciega es inferior al de los otros dos niños del estudio. Esto se debe a que la madre de la niña ciega inicia proporcionalmente muchas más conversaciones que las madres de los otros niños. Esta comparación entre frecuencias y porcentajes de inicios de conversaciones pone de relieve, otra vez, lo que antes he apuntado, que las madres de los niños ciegos son más persistentes y perseverantes en sus intentos de incorporación de sus hijos a las conversaciones; en otras palabras, fomentan más su participación en conversaciones.

Los resultados que hemos hallado con Gina Conti-Ramsden están en desacuerdo con otros relatos anteriores de la contingencia y la participación en los intercambios conversacionales (Kekelis y Andersen, 1984; Kekelis y Printz, 1996). Ello se debe, probablemente, a las diferencias en los niños estudiados, en los contextos (juego con juguetes en el estudio de Kekelis y Prinz frente a interacción libre en nuestro estudio), y en el tema de análisis (respuestas a preguntas y directivos en la investigación de Kekelis y Printz versus todo tipo de interacciones en nuestro estudio). En cualquier caso, los resultados de Kekelis y Prinz (1996) y los nuestros coinciden en la escasez de respuestas no contingentes en los niños ciegos, contrariamente a las sugerencias de Kekelis y Andersen (1984). 
Por tanto, los resultados que hemos encontrado no dan crédito a la idea de que los niños ciegos se parecen a los autistas en su incapacidad para iniciar conversaciones y producir emisiones contingentes para mantener el tema de la conversación.

A partir de lo expuesto se presentan los siguientes comentarios finales. El primero es que los rasgos autistas del lenguaje de los niños ciegos que acabamos de discutir parecen ser el resultado de generalizaciones abusivas que, muchas veces, son extraídas de estudios que no alcanzan los criterios de calidad metodológica deseables. Aunque algunos niños ciegos puedan presentar algún rasgo autista, eso no permite decir que los niños ciegos en su generalidad presenten rasgos semejantes a los de los autistas en su lenguaje, especialmente si tenemos en cuenta la heterogeneidad de la población de niños ciegos. Por otra parte, es posible que los niños ciegos que presentan rasgos autistas también presenten algunas características adicionales, lo cual pondría aún más en cuestión las afirmaciones de Hobson y colegas. De hecho, la mayor parte de los niños ciegos estudiados por Hobson et al., que presentaban rasgos autistas, tenían un cociente intelectual inferior a 70 (Brown et al., 1997; Minter et al., 1998). Por tanto bien se podría pensar que no es la ceguera en sí misma la causante de ciertos síntomas, sino su asociación con otros problemas.

En segundo lugar, aún cuando los niños ciegos presenten comportamientos que son aparentemente similares a los de los niños autistas (como es el caso del uso de las imitaciones y el habla formuláica, o el habla llamada egocéntrica), sin embargo, su significado, las funciones que cubren, y las causas que los provocan son diferentes en ambos grupos de niños. La mera analogía superficial de dos comportamientos no es suficiente para establecer equivalencias entre ellos. Por eso, decir que las niñas ciegas presentan rasgos semejantes a los de los autistas no ayuda mucho a la comprensión de la ceguera o a la comprensión del lenguaje de los niños ciegos, a menos que realicemos análisis más profundos. Por ejemplo, en el caso del habla 
egocéntrica, parece claro que las limitaciones de los niños ciegos para hablar de la realidad externa están más relacionadas con sus dificultades de acceso a la información de lo que ocurre en su entorno que a una incapacidad consustancial al hecho de ser ciego.

Precisamente este ejemplo pone de manifiesto una tercera consideración que me gustaría realizar del desarrollo de los niños ciegos, y que conecta directamente con la polémica sobre su capacidad para formar una teoría de la mente. Los niños ciegos sin trastorno asociado parecen ser extremadamente sensibles a las experiencias educativas y ambientales. En la medida en que la familia y la escuela ayudan a los niños ciegos a superar las restricciones de acceso al mundo externo, su desarrollo puede ser muy positivo. Los niños ciegos que están expuestos a un medio estimulante, se benefician grandemende de él y su desarrollo puede ser excelente, y los rasgos autistas pueden estar ausentes de su lenguaje. Desgraciadamente, sabemos que el efecto de la intervención en los niños autistas es mucho más frustrante. Esta diferencia entre niños ciegos y autistas es la que Hobson no contempla en su teoría, y la que, en mi opinión, demuestra su debilidad. En mi opinión, la necesidad de justificar su teoría de la percepción actitudinal y la relación interpersonal lleva a Hobson a buscar síntomas autistas en los niños ciegos de una manera que no es todo lo rigurosa que se desearía.

Finalmente, y centrándome un poco más en el proceso de adquisición del lenguaje, creo que es necesario superar la visión monolítica sobre su adquisición y desarrollo. Cualquier teoría de la adquisición del lenguaje debe contemplar y explicar la existencia de importantes variaciones en su adquisición. La forma en que los niños ciegos aprenden a hablar es un ejemplo señero de cómo es posible adquirir el lenguaje utilizando recursos y estrategias diferentes a los que, hasta hace muy poco, se consideraron convencionales. Por otra parte, su capacidad para adquirir el lenguaje sin tener información visual sobre la realidad pone en entredicho la teoría de la base sensorial y cognitiva para el aprendizaje del lenguaje, defendida por Dunlea (1989) y Andersen et 
El lenguaje de los niños ciegos y la cuestión de los rasgos autistas

al. (1993); asimismo, pone de manifiesto la necesidad de que las teorías de la adquisición del lenguaje den mayor relieve al trabajo que los niños desarrollan para analizar el lenguaje, que proporciona, en sí mismo, una inestimable fuente de información.

\section{Referencias}

Andersen, E. S., Dunlea, A. y Kekelis, L. S. (1984). Blind children's language: Resolving some dífferences. Journal of Child Language, $11,645-664$.

Andersen, E. S., Dunlea, A. y Kekelis, L. S. (1993). The impact of input: language acquisition in the visually impaired. First Language, 13, 23-49.

Baron-Cohen, S. (1995). Mindblindness. An essay on autisim and theory of mind. Cambridge, MA: The MIT Press.

Barrett, M. (1983). The early acquisition and development of meaning of action-related words. En T. B. Seiler y W. Wannenmacher (Eds.), Concept development and the development of word meaning (pp. 191-209). Berlin: Springer-Verlag.

Brown, R., Hobson, R.P., Lee, A. y Stevenson, J. (1997). Are there "autistic-like" features in congenitally blind children? Journal of Child Psychology and Psychiatry, 38, 693-703.

Burlingham, D. (1965). Some problems of ego development in blind children. The Plychoanalytic Study of the Child, 20, 194-208.

Castro, J. y Pérez-Pereira, M. (1996). Funciones comunicativas del lenguaje de niños ciegos y videntes. Infancia y Aprendizaje, 74, 139-154.

Chiat, S. (1982). If I were you and I were me: The analysis of pronouns in a pronoun-reversing child. Journal of Child Language, 9, 359379.

Conti-Ramsden, G. y Pérez-Pereira, M. (1999). Conversational interactions between mothers and their infants: The case of congenital blindness. Journal of Visual Impairment and Blindness, 93, 691-703. 
Dale, P. y Crain-Thoreson, C. (1993). Pronoun reversals: who, when and why? Journal of Child Language, 20, 573-589.

Dunlea, A. (1984). The relation between concept formation and semantic roles: Some evidence from the blind. En L. Feagans, C. Garvey y R. Golinkoff (Eds.), The origin and growth of communication (pp. 224-243). Norwood: Ablex.

Dunlea, A. (1989). Vision and the emergence of meaning. Blind and sighted cilildren's early language. Cambridge: Cambridge University Press.

Erin, J. N. (1990). Language samples from visually impaired fourand five-year olds. Journal of Childhood Communication Disorders, 13, 181-191.

Fay, W. H. (1973). On the echolalia of the blind and of the autistic child.Journal of Speech and Hearing Disorders, 38, 478-489.

Fraiberg, S. (1977). Insights from the blind. Londres: Souvenir Press.

Fraiberg, S. y Adelson, E. (1973). Self-representation in language and play: Observations of blind children. Psychoanalysis Quarterly, 42, 539-562.

Fraiberg, S. y Freedman, D. A. (1964). Studies in the ego development of the congenitally blind child. The Psychoanalytic Study of the Child, 19, 113-169.

Frith, U. (1989). Autism. Explaining the enigma. Oxford: Blackwell. Happé, F. (1994). Autism: an introduction to psychological theory. Londres: UCL Press.

Hernández-Pína, F. (1984). Teorías sociopsicolingüísticas y su aplicación a la adquisición del español como lengua matema. Madrid: Siglo XXI de España.

Hobson, R. P. (1993a). Autism and the development of mind. Hillsdale: Erlbaum.

Hobson, R. P. (1993b). Through feeling and sight to self and symbol. En U. Neisser (Ed.), Ecological and interpersonal knowledge of self (pp. 254-279). Cambridge: Cambridge University Press.

Hobson, R. P., Brown, R., Minter, M. E. y Lee, A. (1997). Autism revisited: the case of congenital blindness. En V. Lewis y G. M. 
El lenguaje de los niños ciegos y la cuestión de los rasgos autistas

Collis (Eds.), Blindness and psychological development in young children (pp. 99-115). Leicester: British Psychological Society.

Keeler, W. R. (1957). Autistic patterns and defective communication in blind children with retrolental fibroplasia. En P. H. Hoch y J. Zubin (Eds.), Psychopathology of communication (pp. 64-83). Nueva York: Grune and Stratton.

Kekelis, L. S. y Andersen, E. S. (1984). Family communication styles and language development. Journal of Visual Impairment and Blindness, 78, 54-65.

Kekelis, L. S. y Prinz, P. M. (1996). Blind and sighted children with their mothers: the development of discourse skills. Journal of Visual Impairment and Blindness, 90, 423-436.

Kitzinger, M. (1984). The role of repeated and echoed utterances in communication with a blind child. British Journal of Disorders of Communication, 19, 135-146.

Lee, A., Hobson, R. P. y Chiat, S. (1994). I, you, me and autism: An experimental study. Journal of Autism and Developmental Disorders, 24, 155-176.

Lieven, E. V. M., Pine, J. M. y Baldwin, G. (1997). Lexically based learning and early grammatical development. Journal of Child Language, 24, 187-219.

Lord, C. y Paul, R. (1997). Language and communication in autism. En D. J. Cohen y F. R. Volkman (Eds.), Handbook of autism and pervasive developmental disorders (pp. 247-264) (2da. ed.). Nueva York: Wiley.

McCune, L. (1991). Review of "Vision and the emergence of meaning: Blind and sighted children's early language" by Anne Dunlea. Language and Speech, 34, 97-103.

Miecznikowski, A. y Andersen, E. (1986). From forrnulaic to analysed speech: Two systems or one? En J. Connor-Linton, C. J. Hall y M. McGinnis (Eds.), Southern California Occassional Papers in Linguistics, Volume 11: Social and Cognitive Perspectives on Language (pp. 181-202). Los Angeles: University of Southern California. 
Minter, M., Hobson, R. P. y Bishop, M. (1998). Congenital visual impairment and 'theory of mind'. British Journal of Developmental Psychology, 16, 183-196.

Moore, Y. y McConachie, H. (1994). Communication between blind children and severely visually impaired children and their parents. British Journal of Developmental Psychology, 12, 491-502.

Nagera, H. y Colonna, A. B. (1965). Aspects of the contribution of sight to ego and drive development. A comparison of the development of some blind and sighted children. The Psychoanalitic Study of the Child, 20, 267-287.

Norgate, S., Lewis, V. y Collis, G. (3-6 Sept. 1997). Developing the capacity to refer: how the study of blind infants informs theoretical frameworks of lexical development. Ponencia presentada en el VIl European Conference on Developmental Psychology, Rennes, Francia.

Pérez-Pereira, M. (1994). Imitations, repetitions, routines, and the child's analysis of language: insights from the blind. Journal of Child Language, 21, 317-337.

Pérez-Pereira, M. (1999). Deixis, personal reference, and the use of pronouns by blind children. Journal of Child Language, 26, 655-680.

Pérez-Pereira, M. y Castro, J. (1992). Pragmatic functions of blind and sighted children's language: a twin case study. First Language, 12, 17-37.

Pérez-Pereira, M. y Castro, J. (1997). Language acquisition and the compensation of visual deficit: New comparative data on a controversial topic. British Journal of Developmental Psychology, $15,439-459$.

Pérez-Pereira, M. y Conti-Ramsden, G. (1999). Language development and social interaction in blind children. Hove, GB: Psychology Press.

Pérez-Pereira, M. y Conti-Ramsden, G. (en prensa). The role of directives in verbal interactions between blind children and their mothers. Journal of Visual Impairment and Blindness. 
Pérez-Pereira, M., Forján, M. y García, S. (1996). Comparanza da adquisición das formas de posesión do galego e o castelán en nenos bilingües. Cadernos de Lingua, 14, 21-38.

Peters, A. (1987). The role of imitation in the developing syntax of a blind child. Text, 7. 289-311.

Peters, A. (1994). The interdependence of social, cognitive, and linguistic development: evidence from a visually impaired child.En H. Tager-Flusherg (Ed.), Constraints on language acquisition: Studies of atypical children (pp. 195-220). Hillsdale, NJ: Erlbaum.

Peters, A. (12-16 Julio, 1999). The development of reference to self and other by one blind child. Comunicación presentada en el VIII International Congress for the Study of Child Language (IASCL), San Sebastián.

Pine, J. M. y Lieven, E. V. (1993). Reanalysing rote-leaned phrases: individual differences in the transition to multiword speech. Jornal of Child Language, 20, 551-571.

Shum, G., Conde, A. y Díaz, C. (1992). Pautas de adquisición y uso del pronombre personal en la lengua española. Un estudio longitudinal. Estudios de Psicología, 48, 67-86.

Snow, C. (1993). Saying it again: the role of expanded and deferred imitations in language acquisition. En K. E. Nelson (Ed.), Children's language (pp. 29-58) (Vol 4). Hillsdale: Erlbaum.

Speidel, G. E. (1989) Imitation: a bootstrap for learning to speak? En G. E. Speidel y K. E. Nelson (Eds.), The many faces of imitation in language learning (pp.151-179). Berlin: Springer-Verlag.

Tager-Flusberg, H. y Calkins, S. (1990). Does imitation facilitate the acquisition of grammar? Evidence from a study of autistic, Down's syndrome and normal children. Journal of Child Language, 17, 591-606.

Urwin, C. (1978). The development of communication between blind infants and their mothers. En A. Lock (Ed.), Action gesture and symbol: the emergence of language (pp.79-108). Londres: Academic Press. 
Urwin, C. (1984a). Communication in infancy and the emergence of language in blind children. En R. L. Schieffelbuch y J. Pickar (Eds.), The acquisition of communicative competence (pp.191209). Baltimore: University Park Press.

Urwin, C. (1984b). Language for absent things: leaning from visually handicapped children. Topics in Language Disorders, 4, 24-37.

Warren, D. F. (1994). Blindness and children. An individual differences approach. Nueva York: Cambridge University Press.

Webster, A. y Roe, J. (1998). Children with visual impairments. Social interaction, language and teaching. Londres: Routledge.

Willis, D. M. (1979). Early speech development in blind children. The Psychoanalytic Study of the Child, 34, 85-117.

Wilson, B. y Peters, A. M. (1988). What are you cookin' on a hot?: Movement constraints in the speech of a three-year-old blind child. Language, 64, 249-273. 